\title{
Local Stress Measurement Using the
}

\section{Thermoelastic Effect}

\author{
A method for determining local stresses associated \\ with stress concentrations by experimental measurement \\ of temperature changes due to elastic deformation \\ is described and experimentally tested
}

\author{
by R. W. Dunlap, E. E. Hucke and D. V. Ragone
}

\begin{abstract}
A technique for measuring local stresses in metallic specimens is proposed and tested. The technique depends on the experimental measurement of temperature changes in stressed members due to adiabatic elastic deformation. At a free boundary in a body under plane stress, these temperature changes are directly related to the value of the tangential principal stress. The technique is suited for measurement of stress-concentration effects, since the temperature changes can be measured with thermocouples featuring extremely small junctions.

A simple stress-concentration geometry, the finitewidth strip with a central circular hole, is chosen as a model system for this study. Heat transfer in this geometry due to the temperature gradients produced by elastic deformation is analyzed. It is shown that the ratio of the temperature change at a reference section to the change at the locale of the stress concentration can be used to determine the stress-concentration factor, allowing for heat-transfer effects. An experimental measurement system capable of obtaining reproducible results with the thermal-measurement technique is described, and experimental results are given for the model geometry which agree favorably with theoretical predictions. Application of the technique to other problems is discussed.
\end{abstract}

\section{List of Symbols}

$$
\begin{aligned}
C_{P} & =\text { heat capacity at constant pressure } \\
D & =\text { hole diameter } \\
F & =\text { loading function } \\
\dot{F} & =\text { time derivative of loading function }
\end{aligned}
$$

\footnotetext{
R. W. Dunlap and D. V. Ragone are Staff Associate and Assistant Director, of the Laboratory, respectively, General Atomic Division, General Dynamics, San Diego, Calif. E. E. Hucke is Professor of Metallurgical Engineering, University of Michigan, Ann Arbor, Mich.

Paper was presented at the 1967 SESA Spring Meeting held in Ottawa, Ont., Can., on May 16-19.

Taken in part from dissertation of R. W. Dunlap, submitted in partial fulfilment of the requirements for degree of Doctor of Philosophy at the
} University of Michigan, Ann Arbor, Mich.

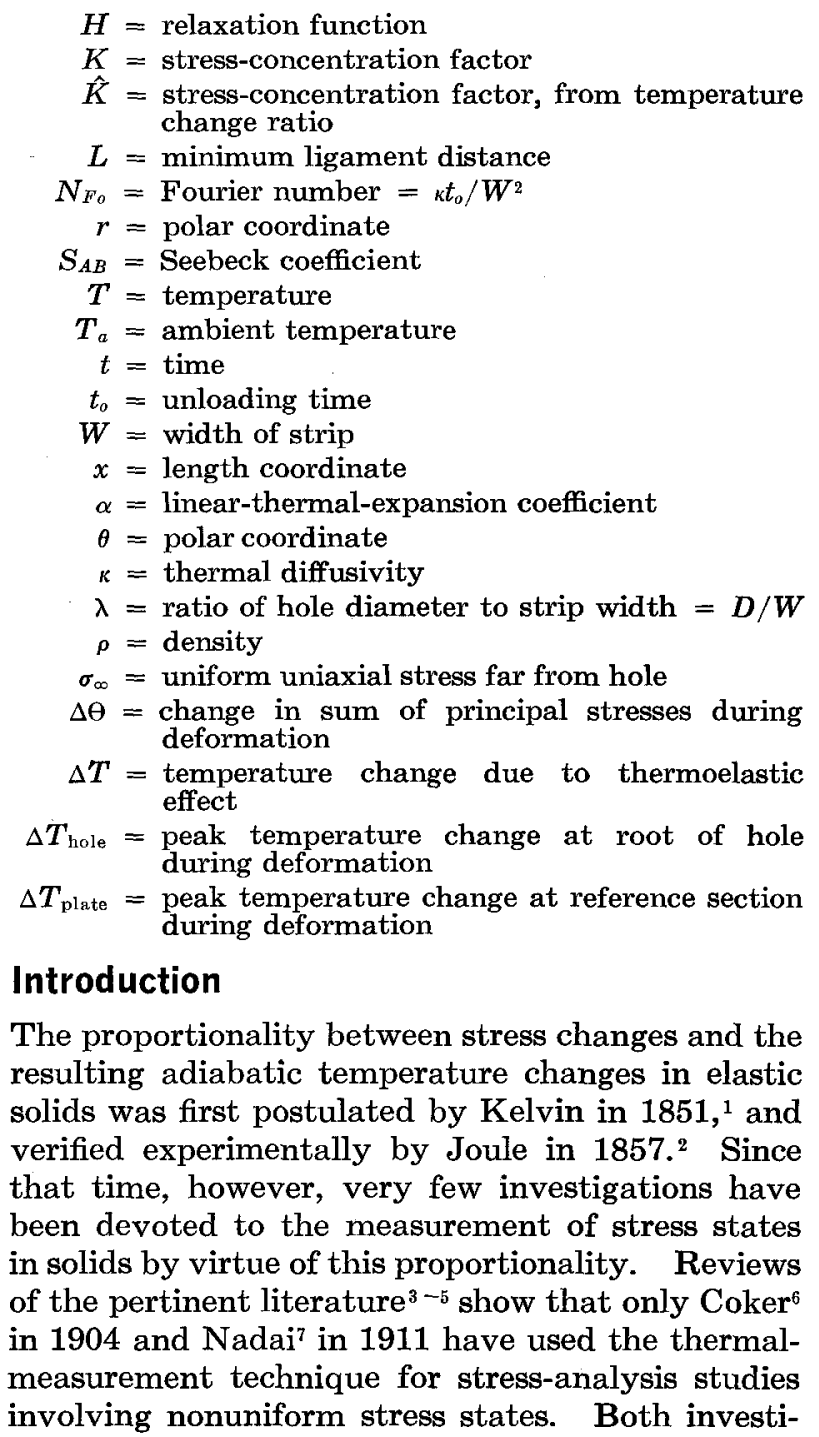




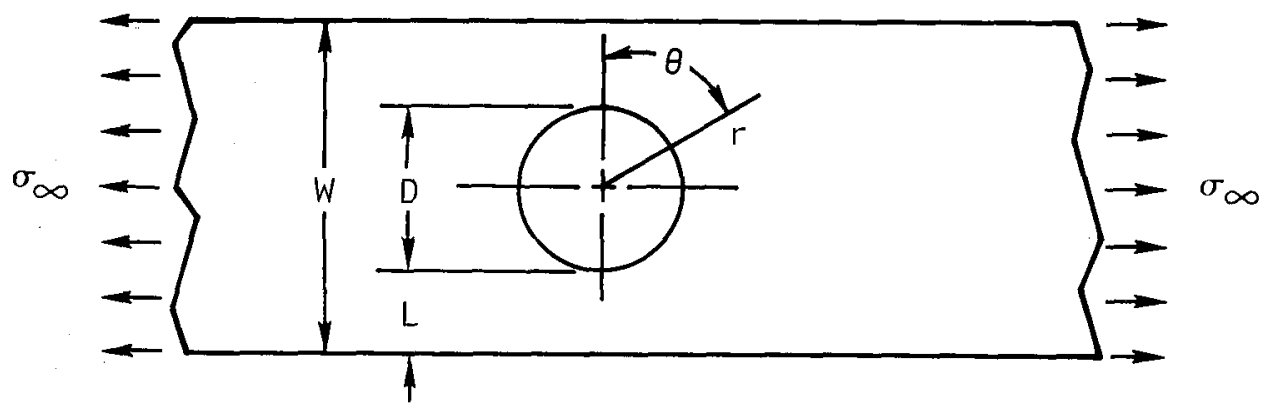

Fig. 1-Geometry, coordinate system and parameters involved in the model system

gators analyzed the bending stress over the cross section of a steel beam, using a large cross-section size to minimize the temperature gradients. A linear distribution of stress over the cross section was verified in each study. Quite recently, Dillon and Tauchert have measured thermoelastic temperature changes as part of a more general study of the heat generated during small plastic deformations of metals. ${ }^{8}$ Their study included measurement of temperature changes due to the elastic bending of a cantilever beam, but the results obtained were qualitative in nature.

Practical application of the technique up to this time has been limited for two reasons. The small temperature changes involved, typically $0.1-0.4^{\circ} \mathrm{C}$ for most metals at elastic-limit stress levels, lead to instrumentation and measurement problems. Also, if nonuniform stress states are studied, temperature differences produced by deformation are dissipated by conductive heat transfer in the stressed member, in times which can be smaller than the characteristic response times of temperature-measurement transducers.

The present study was undertaken to investigate the limitations and potentialities inherent in the thermal technique, particularly for studies of nonuniform stress states. An advantage of the method is that temperature changes can be measured with thermocouples featuring extremely small junctions. Hence, the technique would appear to be suited for measurement of local stresses associated with various stress-concentration geometries. For this study, a typical stress-concentration geometry, involving the tensile loading of a finite-width strip containing a central circular hole, is taken as a model system (Fig. 1). Since a theoretical solution for the stress state in the neighborhood of the hole is available, the temperature distribution produced by elastic deformation and the changes in this temperature distribution due to conductive heat transfer can be calculated.

An analytical study based on such considerations is made. The Duhamel integral is used to predict the temperature change occurring at the locale of the maximum stress concentration for an arbitrary loading schedule. It is shown that the measurement of a temperature change ratio can be used to determine the stress-concentration factor for the geometry, regardless of heat-transfer effects. Experimental methods and procedures used to measure thermoelastic temperature changes are then discussed, and experimental results for the model problem are given. Specimen materials used in the experimentation include stainless steel, brass and aluminum alloys, and thermocouple voltages measured are on the order of ten microvolts. The extension of the experimental techniques used for the analysis of the model geometry and the application of the thermal-measurement technique to other studies is discussed.

\section{Analytical Studies}

The stress state in the model geometry, in the annular region with outer diameter $\mathrm{W}$ surrounding the hole, has been investigated analytically by Howland.9, 10 The mathematical solution has been verified experimentally, ${ }^{11}$ and the geometry has served as a standard for several experimental stressanalysis investigations. ${ }^{12,13}$

The peak stress in the geometry occurs at the root of the hole, the location $(r, \theta)=(D / 2,0)$. For this study, the stress-concentration factor for the geometry is defined as the value of the peak stress divided by the value of the nominal stress $\sigma_{\infty}$ far from the hole. (The stress $\sigma_{\infty}$ will be referred to as the plate stress.) The stress-concentration factor defined in this way is 3.03 for $\lambda=0.1$, and 4.32 for $\lambda=0.5$.

The stress distributions involved for the two $\lambda$ values, 0.1 and 0.5 , differ. Using Neuber's terminology, ${ }^{14}$ the case $\lambda=0.1$ is an example of a shallow notch problem, in which the effect of the stress concentration is localized around the notch. The case $\lambda=0.5$ is an example of the deep notch where the disturbance of stress is localized over the minimum section. Heat transfer due to stress-induced temperature gradients in the first case is two dimensional in nature; for the second case, heat transfer may be approximated by a one dimensional problem. The analysis below indicates that the temperature-measurement technique may be used in either case to determine the stress-concentration factor for the geometry.

\section{Heat-transfer Effects}

The thermoelastic temperature change for an incremental volume element in an isotropic solid undergoing an adiabatic, elastic deformation is ${ }^{5,15}$

$$
\Delta T=-\left(T_{a} \alpha / \rho C_{p}\right) \Delta \theta
$$

The temperature change is associated with the dilation produced by the deformation. For mate- 
Fig. 2-Geometry and boundary-value problem involved in two-dimensional heat-transfer approximation

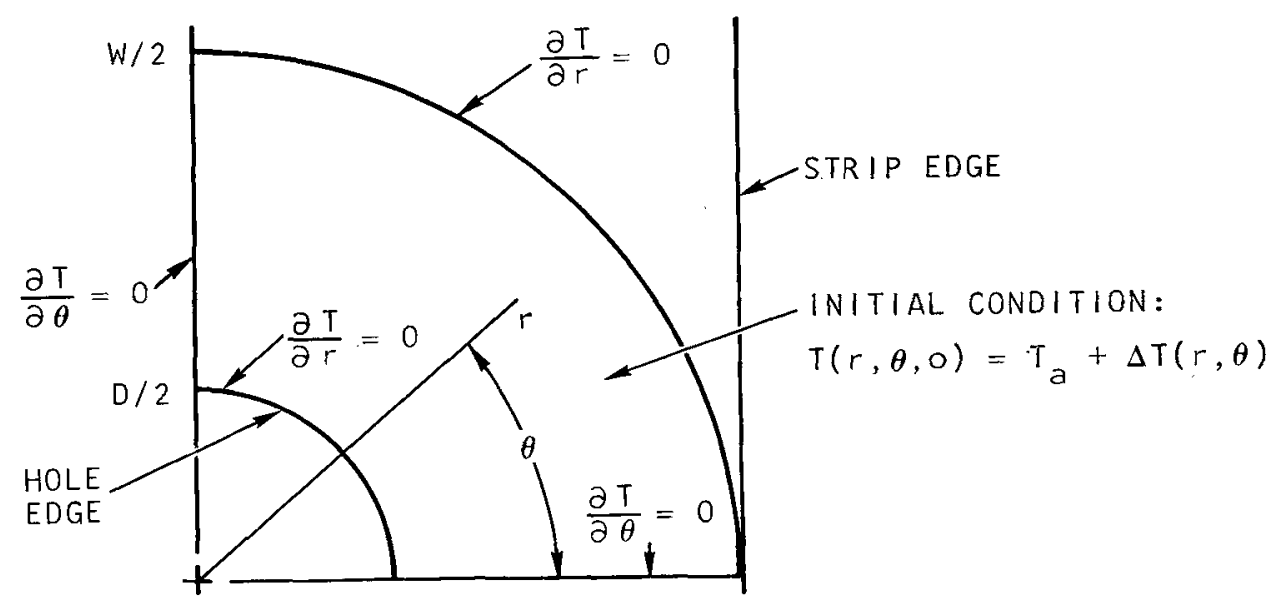

BOUNDARY VALUE PROBLEM:

$$
\begin{array}{rlr}
T_{t}(r, \theta, t)= & K\left[T_{r r}(r, \theta, t)+r^{-1} T_{r}(r, \theta, t)+r^{-2} T_{\theta \theta}(r, \theta, t)\right], \\
& \text { FOR }(D / 2<r<W / 2,0<\theta<\pi / 2, \quad t>0) \\
T(r, \theta, o)=T_{a}+\Delta T(r, \theta), \quad(D / 2<r<W / 2,0<\theta<\pi / 2) & (t>0) \\
T_{r}(D / 2, \theta, t)=T_{r}(W / 2, \theta, t), & (t>0) \\
T_{\theta}(r, o, t)=T_{\theta}(r, \pi / 2, t), &
\end{array}
$$

rials with a positive thermal-expansion coefficient, simple tensile stressing is accompanied by a temperature drop; simple compressive stressing, a temperature rise; and pure shear, no temperature change.

If the model geometry is subjected to an instantaneous uniaxial loading (from stress zero to stress $\sigma_{\infty}$ far from the hole), the instantaneous temperature change at each point is given by eq (1). At locations far from the hole, the temperature change is uniform over the cross section, with $\Delta \theta=\sigma_{\infty}$. At the edge of the hole, the radial stress is zero, and the initial thermoelastic temperature change is proportional to the tangential stress at each point. In the neighborhood of the hole, the sum of principal stresses is a steep function of position, and the deformation creates steep temperature gradients. Hence, the instantaneous temperature changes at the hole edge are quickly dissipated by conductive heat tranisfer.

Ignoring the small loss due to convective heat transfer from specimen surfaces to the air, heattransfer effects at the root of the hole can be studied with the boundary-value problems shown in Figs.
Fig. 3-Geometry and boundary-value problem involved in one-dimensional:heat-transfer approximation

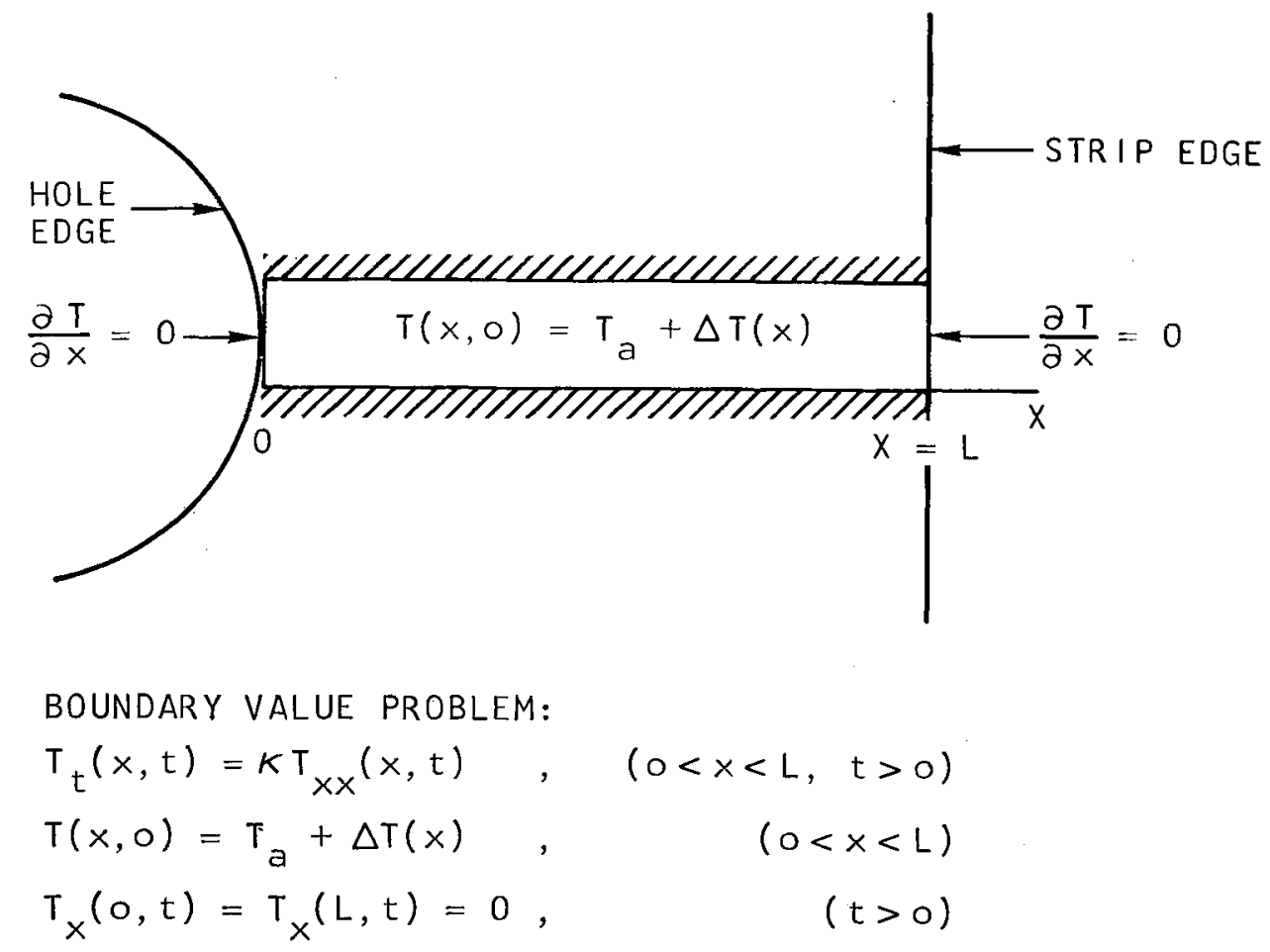




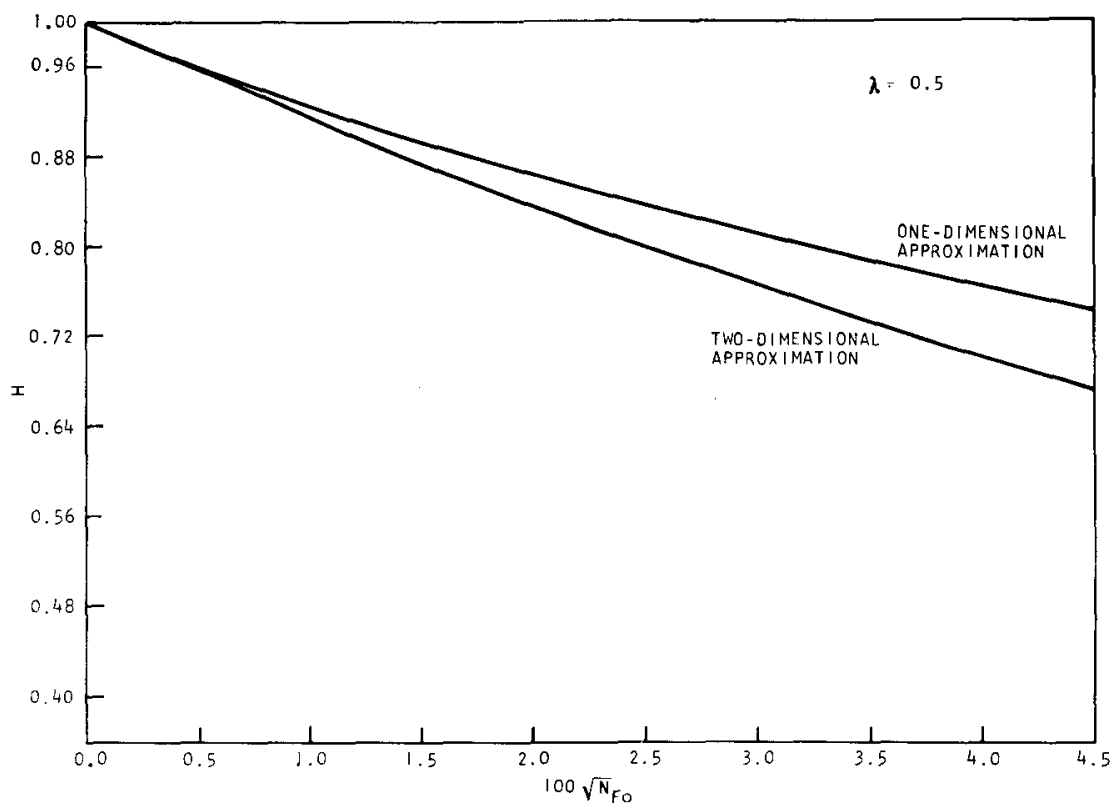

Fig. 4-Relaxation function calculated from approximate heat-transfer models $(\lambda=0.5)$

2 and 3. In the two-dimensional problem (Fig. 2), heat conduction in the entire region surrounding the hole is accounted for. From symmetry, only one quadrant of the geometry need be considered. The angular boundary conditions result from this symmetry, while the radial condition at the hole is based on the absence of convective heat transfer. The radial boundary condition at $r=W / 2$ is an approximation to the true physical situation, but analysis of steady-state temperatures indicates it is a good approximation. The one dimensional problem (Fig. 3) is an approximation which is applicable when heat transfer from the root of the hole is dominated by the temperature gradient across the minimum section.

Both problems can be solved, utilizing Howland's results and eq (1) to compute the initial temperature distribution. Details of the solutions are given elsewhere, ${ }^{5}$ and only the pertinent results are shown here. The relaxation of temperature at the root of the hole due to heat conduction is represented by a relaxation function:

$$
\Delta T(t) / \Delta T(0)=H\left(\lambda, N_{F o}\right)
$$

This is the ratio of the temperature difference from the ambient at any time $t$ to the initial temperature difference; each temperature difference is evaluated at the root of the hole. The relaxation function is a generalized dimensionless temperature, and is a function of $\lambda$, the dimensionless geometry parameter, and $N_{F o}$, the Fourier number, or dimensionless time. Figures 4 and 5 show values of this relaxation function computed from results of both the one- and two-dimensional analyses. Results for $\lambda=0.5$ (Fig. 4) indicate that the one-dimensional heat-transfer model is a good approximation for small times. Results for $\lambda=0.1$ (Fig. 5) show that the one-dimensional approximation is inade- quate, and the heat-conduction problem involved in this shallow notch case is always two-dimensional in nature.

Temperature relaxation occurs rapidly at the hole. For example, if a 1-in.-wide 6061 aluminum specimen with $1 / 2$-in.-diam hole were instantaneously stressed, Fig. 4 shows that $10 \mathrm{msec}$ later (corresponding to $100 \sqrt{N_{F o}}=3.24$ ), only about 80 percent of the initial $\Delta T$ would exist at the root of the hole. Since the heat-transfer effects demonstrated here can occur in such short characteristic times, both the effect of finite loading time and the effect of thermocouple response time on the temperature changes are important.

\section{Loading-time Effects}

The relaxation of temperature at the root of the hole is described by eq (2) for a step-function loading process. The Duhamel integral is used to give the relaxation for an arbitrary loading process in terms of the relaxation for a step function loading:

$\Delta T(t)=\Delta T(\mathbf{0}) H(t)+\int_{0}^{t} \dot{F}(\tau) H(t-\tau) d \tau$

Here, $F(\tau)$ is the loading function, defined as the value of the total $\Delta T$ developed at the root of the hole up to time $\tau$, in the absence of heat-transfer effects. The loading function can be computed from eq (1) and a knowledge of the load-time relationship. Equation (3) simplifies to eq (2) for the case of step-function loading.

Two assumptions are involved in the use of this equation to determine temperature changes developed under arbitrary loading procedures. First, the loading rate must be rapid enough that heattransfer effects other than conduction near the hole are negligible. Second, heat-generation effects due 
Fig. 5-Relaxation function calculated from approximate heat-transfer models! $(\lambda=0.1)$

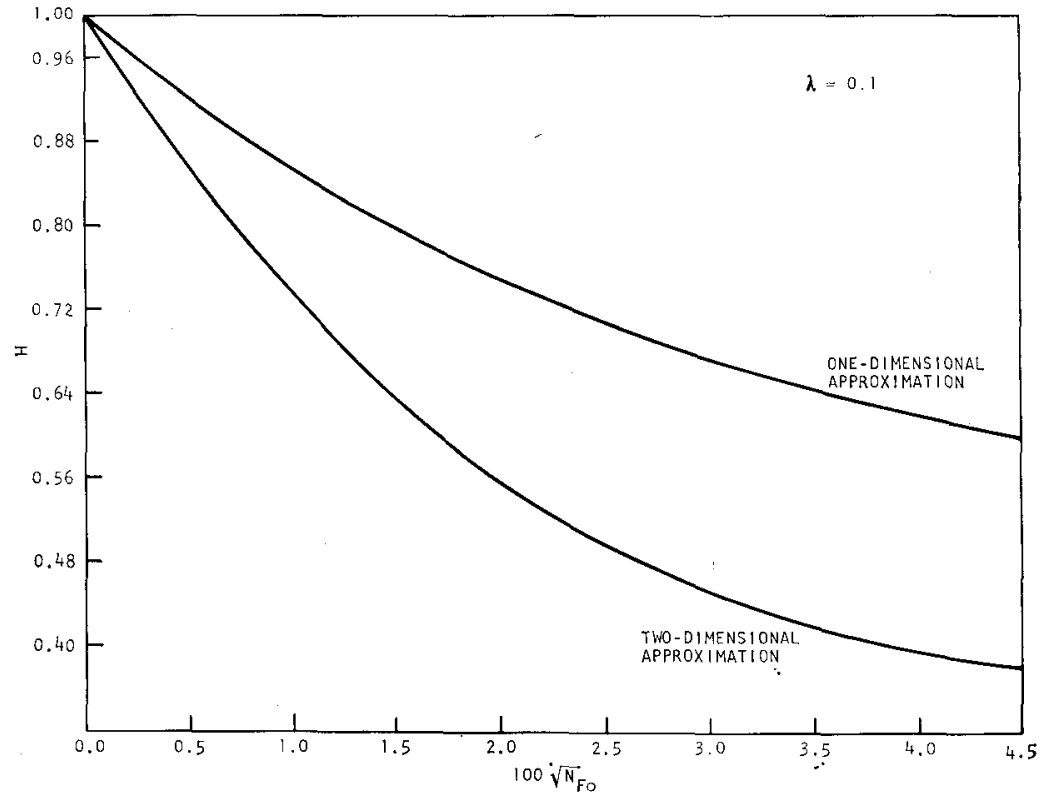

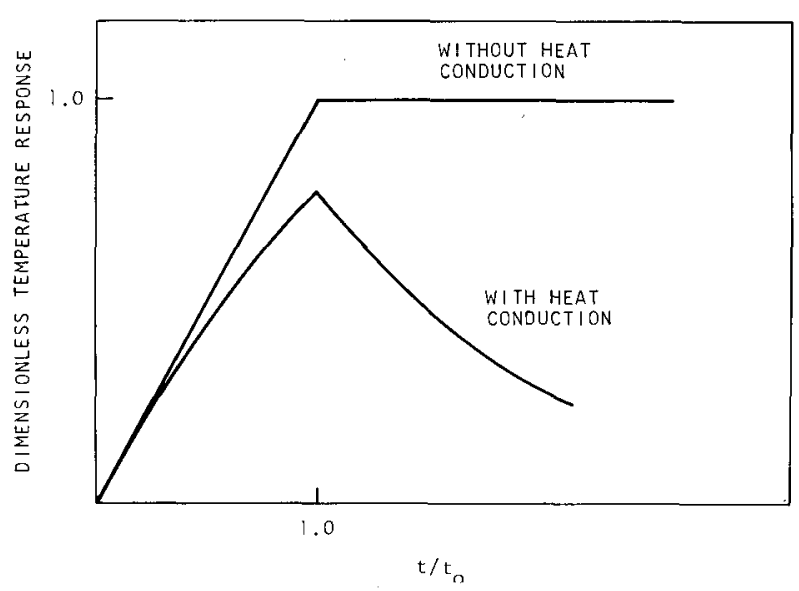

Fig. 6-Schematic temperature-response curves for linear loading, showing effect of heat conduction

to thermoelastic dissipation must be negligible, as they are not accounted for in eq (1). The latter assumption rules out the use of eq (3) to study the steady-state temperature changes involved in an oscillatory loading. Biot ${ }^{16}$ has noted the secondorder nature of the heat generation and resulting temperature changes due to thermoelastic dissipation. This second-order heat becomes important if it accumulates over a long-time period, as in an oscillatory loading schedule.

Under these restrictions, eq (3) may be used to determine temperature changes occurring at the root of the hole. The solution to this equation is afforded by Laplace transform techniques, as the Laplace transform convolution integral is involved in the equation. Calculations are shown in the Appendix for the case in which the loading function varies linearly with time up to time $t_{0}$, and then remains constant. This is the case of linear loading, an idealization of the loading pattern obtained in many experimental situations. For this case, eq (A-3) governs the relaxation process, and temperature response curves are shown in Fig. 6. Here, the temperature changes occurring at the root of the hole, both with and without heat conduction, are shown schematically. Both curves are normalized with respect to the maximum temperature change occurring in the absence of heat conduction. The peak temperature change which actually obtains in an experimental situation is always less than this maximum value, and occurs at time $t_{0}$, as shown in the bottom curve.

\section{Technique to Determine Stress-concentration Factor}

The peak temperature changes which occur during linear loadings are particularly suited for experimental measurement, and form the basis of a technique to determine the stress-concentration factor for the geometry with temperature measurements.

A stress-concentration factor based on temperature measurements is defined as the ratio of the peak temperature changes occurring at hole and plate.

$$
\hat{K}=\Delta T_{\text {hole }} / \Delta T_{\text {plate }}
$$

This equation is applicable to any stress-concentration geometry, comparing the temperature change at a discontinuity to the temperature change at a reference section. For the model geometry, the peak temperature change at the root of the hole is given by eq (A-4), and the peak change far from the hole is given by eq (A-5). It follows that

$$
\hat{K}=K t_{o}^{-1} \int_{o}^{t_{o}} H(t) d t
$$

where $K$ is the stress-concentration factor for the geometry. Hence, $\hat{K}$ is a dimensionless quantity, a function of the three dimensionless variables $\lambda, K$ and $N_{F o}$.

This equation can be evaluated for the model 


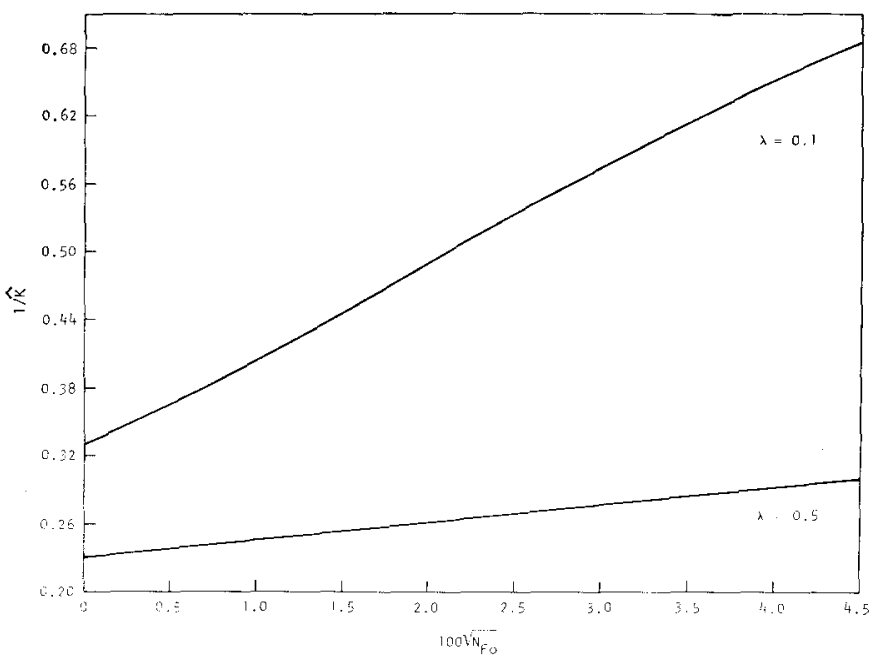

Fig. 7-Analytical results, showing relationship between variables used in thermal-measurement technique

geometry, using the previous two-dimensional approximation to the relaxation function. The results of the evaluation are shown in Fig. 7, for the two cases $\lambda=0.1$ and $\lambda=0.5$. In each case, the relationship between $1 / \hat{K}$ and $N_{F_{0}}{ }^{1 / 2}$ is nearly linear. The intercept of each curve is $1 / K$, while the slope of each curve is related to the stress and resulting temperature gradients involved in the problem. For a problem involving no heat transfer, a line with zero slope is obtained.

The technique for determining the stress-concentration factor from thermal measurements consists in making a parametric study of the temperature changes during linear loading, computing $\hat{K}$ for each data point by eq (4), and plotting $1 / \hat{K}$ values vs. values of the square root of the Fourier number for the geometry. The inverse of the stress-concentration factor is the intercept of this plot.

\section{Upper and Lower Bounds}

An experimental verification of the technique is based on data obtained for the model geometry, for the case $\lambda=0.5$. Based on the analytical model, upper and lower bounds for the experimental data can be established. These bounds refer to lines on the plot of $1 / \hat{K}$ vs. $N_{F_{0}}{ }^{1 / 2}$, between which valid experimental data should lie.

A lower bound for the data is the line for $\lambda=0.5$ shown in Fig. 7. This line, based on the twodimensional heat-transfer approximation discussed above, underestimates the amount of heat conduction which occurs, especially at large times. Therefore, valid experimental data will lie on or above this line.

An upper bound for the data can be based on an examination of eq (5). The relaxation function, Fig. 4, is monotone decreasing and it follows that

$$
\int_{0}^{t_{o}} H(t) d t>t_{0} H\left(t_{0}\right)
$$

From eq (6) and eq (5), an upper bound for the data follows:

$$
1 / \hat{K}<\left[K H\left(t_{o}\right)\right]^{-1}
$$

The upper bound can be evaluated from a knowledge of the two-dimensional relaxation function. Both bounds are shown in Fig. 12, where the experimental data are also shown.

\section{Experimental Equipment and Procedures}

Experimental measurement of thermoelastic temperature changes depends on careful experimental technique. Methods used to load test specimens and measure the temperature changes produced are summarized here. Further details concerning the experimentation are found elsewhere. ${ }^{5}$

\section{Loading System}

A standard guillotine-type loading frame is employed, in which the specimen is axially loaded (Fig. 8). The system is designed to produce a change in stress by unloading. This ensures elastic response of the specimen, and allows easy adjustment of the unloading time. The specimen is loaded by suspending weights from a quick-release magnet at one end of a beam.

The unloading time has to be small enough to preclude excessive signal losses due to thermal relaxation at the hole, and large enough to ensure quasi-static loading. The unloading time is a function of the magnet-energizing-circuit parameters (Fig. 10), and can be varied through a two to one ratio for a given load. Unloading times used in the experimental program varied between 7 and 20 msec. A typical strain-gage trace, showing the linear approximation to the unloading curve, is given in Fig. 11.

To unload the specimen, the magnet is de-

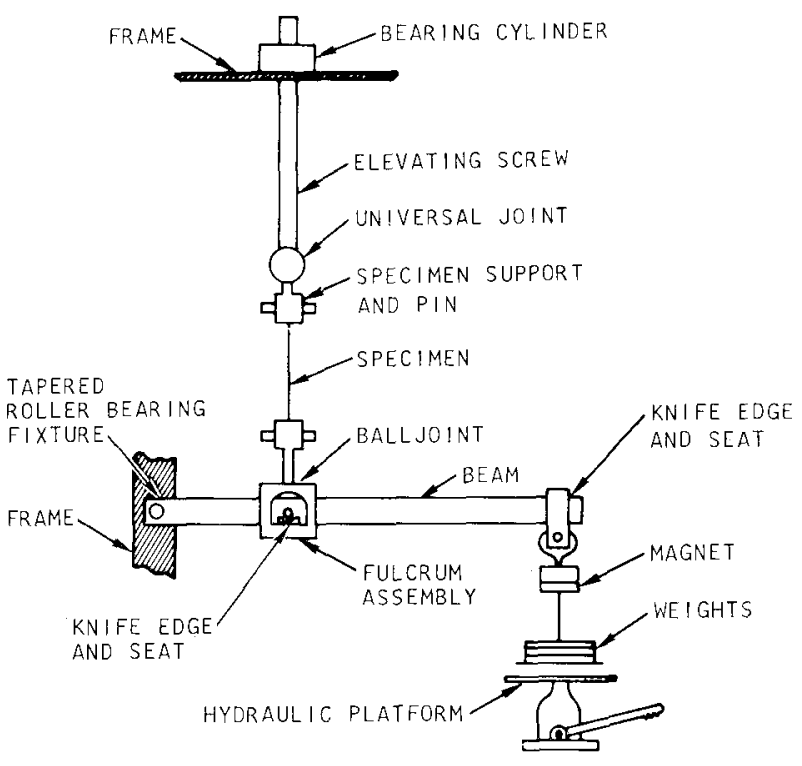

Fig. 8-Schematic diagram of loading apparatus 
Fig. 9-Specifications for strip specimen with central hole
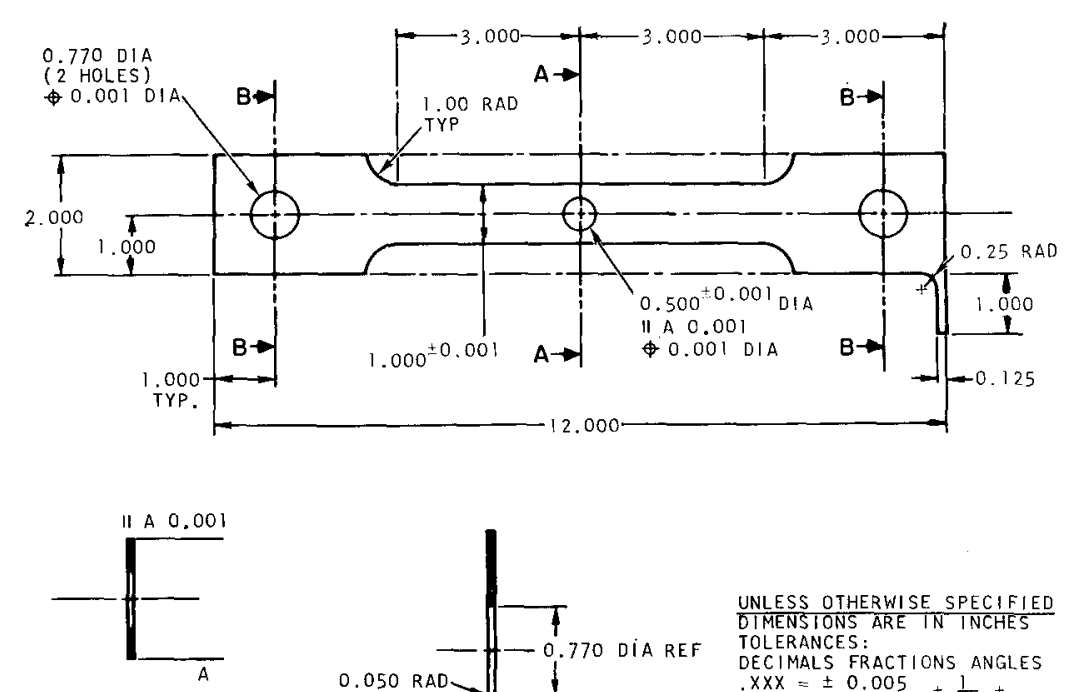

SECTION A-A

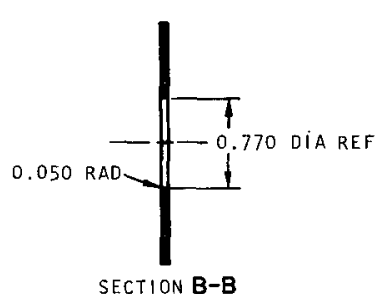

SECTION B-B
UNLESS OTHERWISE SPECIFIED DIMENSIONS ARE IN INCHES TOLERANCES:
DECIMALS FRACTIONS ANGLES $X X X= \pm 0.005 \pm \frac{1}{64} \pm$
$X X= \pm 0.01$

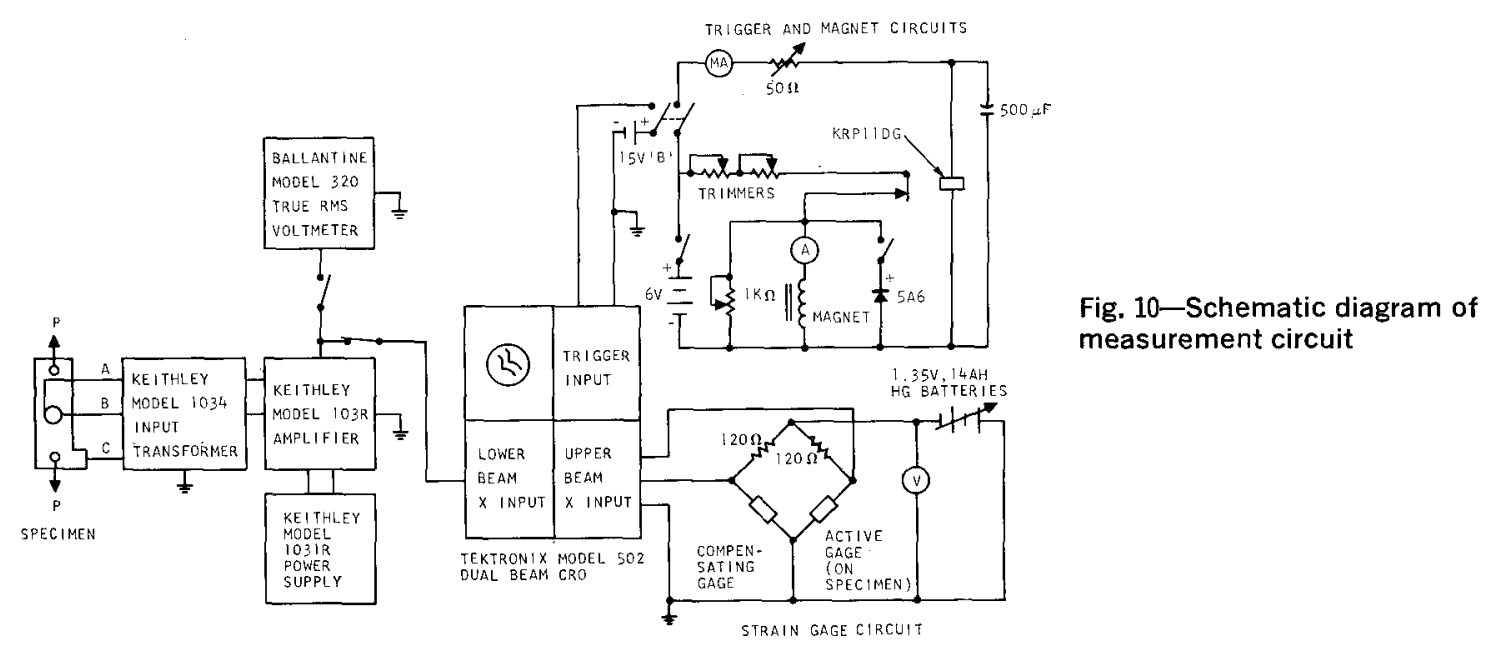

energized by actuating a DPST microswitch. This switch closes a relay circuit after an adjustable time delay, thereby initiating the unloading cycle. Simultaneously, the CRO traces are triggered. Upon unloading, the beam is free to rotate in the vertical plane. Weights, weight pan and rod, and lower magnet platen fall on a hydraulic platform and can be raised into position to prepare for a subsequent unloading cycle.

A typical specimen design is shown in Fig. 9. Specimens are designed with a sufficient lengthwidth ratio to ensure that a uniform uniaxial stress state exists in a large region on either side of the central hole. The extent of these regions is sufficient to ensure that the signal from a thermocouple mounted here is unaffected by axial heat conduction. Bending effects are minimized by the use of pin loading and specification of close dimensional tolerances for the specimens. Specimen pin holes are $0.020 \mathrm{in}$. oversize, and radiused in the thickness dimension. Hence, the specimen is free to rotate about the pin both in and perpendicular to the plane of the specimen, to maintain axial loading. The specimen is prestrained with several loadunload cycles at a stress level slightly higher than the level used for subsequent measurements.

\section{Measurement System}

The measurement system is shown in Fig. 10. For each unloading cycle, a strain-gage signal and a thermocouple signal are simultaneously monitored.

A strain gage mounted at one plate location on the specimen is used to provide a reference signal to which the thermocouple signal can be compared. Foil-type strain gages are used, and a conventional Wheatstone bridge circuit with $d$-c coupling to the CRO is employed.

The thermocouple signal measurement circuit features a commercial a-c low-noise amplifier and external impedance matching input transformer. System gain is $\sim 55,000$, and input resistance 30 ohms. System noise referred to input for bandwidth $10-1000 \mathrm{~Hz}$ is $0.04 \mu \mathrm{v} \mathrm{rms}$, and is monitored with a commercial true rms voltmeter. 


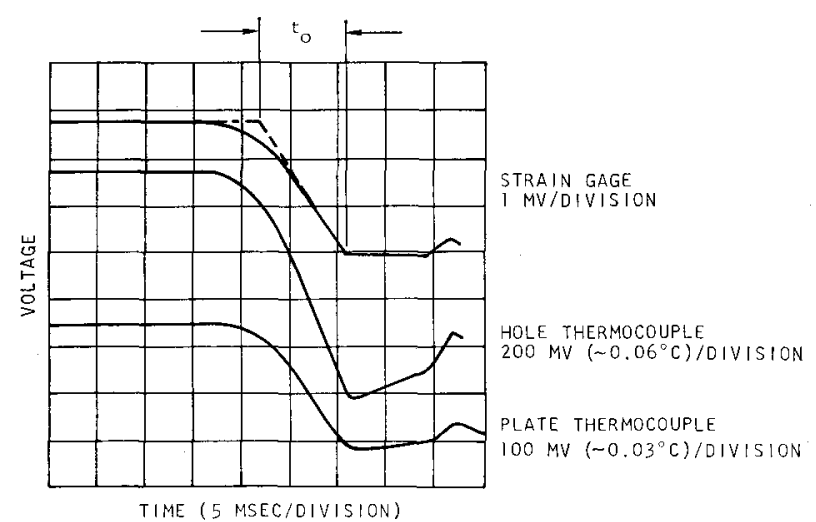

Fig. 11-Strain gage and thermocouple signals for 70-30 brass specimen, showing linear approximation to unloading curve

Thermocouple signal levels range from 3 to 20 $\mu \mathrm{v}$, and achievement of a good signal-to-noise ratio depends on careful use of shielding, grounding and isolation techniques. Optimum results are obtained when the specimen is electrically isolated from the loading frame, the magnet circuit is electrically isolated from the other measurement-system circuits, and electromagnetic and electrostatic shielding is used in the input circuits. The standard deviation for a thermocouple signal, measured in a number of successive unloading cycles, is always less than 3 percent, and is typically 1-2 percent.

\section{Thermocouple Characteristics}

A differential input, intrinsic thermocouple is used for the measurement. One lead, wire C in Fig. 11, is attached to the specimen at an isothermal junction. The other leads are single, bare thermocouple wires welded separately to the specimen at either plate or hole locations. A resistance-welding technique is employed, using a commercial d-c storedenergy welding power supply and handpiece, and a micromanipulator to position the wire. Wires are welded flat to the specimen surface or hole edge, and a typical weld ( 5 mil wire) is oval in cross section, with maximum width 8 mils and length 15 mils. Surface and wire damage is minimized with proper welding procedure, and metallographic cross sections of the welds reveal typical surface damage less than 0.5-1.0-mils deep.

The temperature-change measurements are markedly affected by the response characteristics of the thermocouples. Experiments with thermocouplewire diameters ranging from one to 10 mils, welded at various energy levels, show the effects of variable thermocouple response. ${ }^{5}$ Poor response leads to signal attenuation and the presence of a large lag time between the end of unloading denoted by the strain signal, and the time at which the thermocouple signal reaches maximum deflection. These effects are minimized for the smallest wire size investigated, and minimal welding energy. However, the response characteristics of these small-wire thermocouples are not reproducible, and all data are taken using 5-mil-diam, Chromel and Constan- tan wires. Although these larger-wire thermocouples have slower response, results between different thermocouple installations are reproducible. To ensure identical response characteristics for the hole and plate thermocouples, the lag times associated with each couple are compared. Valid data are obtained only when the lag time associated with the hole thermocouple is less than the lag time associated with the plate thermocouple, under identical unloading conditions. This criterion is demanded because of the effect of heat transfer in the specimen at the hole, and is discussed fully elsewhere. ${ }^{5}$

\section{Experimental Procedure}

The amplifier responds to an attenuated signal $S_{A B} \Delta T$, where $S_{A B}$ is the Seebeck coefficient of the thermocouple $\mathrm{A}-\mathrm{B}$, and $\Delta T$ is the thermoelastic temperature change. The degree of signal attenuation depends on the combined response characteristics of the thermocouple-amplifier system. All measurements are made with an amplifier bandwidth $0.5-1200 \mathrm{~Hz}$, but the upper frequency limit of the combined system is typically set by the thermocouple response characteristics. If the combined system response is identical for measurements made at plate and hole locations, and if identical thermocouple materials are used for the two measurements, the ratio of the voltage measurements yields $\hat{K}$, by eq (4).

Comparison of the voltage levels is made at the end of unloading, where the strain-gage signal exhibits a discontinuity in slope. Signal-amplitude measurements at this point are preferable to measurements of peak deflections because of small fluctuations in stress level which occur after unloading. The thermocouple-signal amplitudes are measured at different oscilloscope sensitivities, and plate and hole signal deflections average 2 and $4 \mathrm{~cm}$ respectively on the CRO screen. Twelve unloading cycles are usually performed for each thermocouple location, and a mean value and standard deviation for the deflection are calculated. Results are then corrected for the effect of circuit loading due to the low input impedance of the amplifying system, and $1 / \hat{K}$ values and associated standard deviations are calculated from eq (4).

\section{Experimental Results}

Typical strain-gage and thermocouple traces for a $70-30$ brass specimen are shown in Fig. 11. Measurements are made with thermocouple polarity reversed, so that strain and temperature deflections have the same sign. The traces from two unloading cycles are superposed, hence the strain gage and hole and plate thermocouple signals are shown simultaneously. The curves shown are copied directly from Polaroid print records. The effect of thermocouple response is seen in the lag-time phenomena previously discussed. The hole-thermocouple lag time shown here is less than the plate- 


\begin{tabular}{|c|c|c|c|c|c|c|}
\hline Material & Width(in.) & $\kappa\left(\mathrm{cm}^{2} / \mathrm{sec}\right)$ & $t_{o}(\mathrm{msec})$ & $100 \sqrt{ } \mathrm{N}_{\mathrm{Fo}}$ & $\Delta \sigma_{\infty}(\mathrm{psi})$ & $1 / \hat{K}$ \\
\hline 321 S.S. & 2.00 & 0.041 & $7.25 \pm 0.25$ & 0.34 & 9400 & $0.245^{*}$ \\
\hline 321 S.S. & 1.75 & 0.041 & $7.25 \pm 0.25$ & $0.39 \pm 0.01$ & 9200 & $0.239=0.008$ \\
\hline 321 S.S. & 1.25 & 0.041 & $8.0 \pm 0.3$ & 0.57 & 9300 & $0.267 *$ \\
\hline 304 S.S. & 1.00 & 0.041 & $9.5 \pm 0.5$ & $0.78 \pm 0.02$ & 8000 & $0.251 \pm 0.008$ \\
\hline $70-30$ Brass & 1.00 & 0.368 & $9.5 \pm 0.25$ & $2.33 \pm 0.07$ & 7700 & $0.276 \pm 0.006$ \\
\hline 6061-T6 Al & 1.00 & 0.676 & $12.0 \pm 0.3$ & $3.55 \pm 0.10$ & 5200 & $0.304 \neq 0.005$ \\
\hline 6061-T6 Al & 1.00 & 0.676 & $20.0 \pm 0.5$ & $4.58 \pm 0.13$ & 3700 & $0.328 \pm 0.008$ \\
\hline
\end{tabular}

* Hole and plate thermocouples have unmatched response characteristics, as shown by lag times.

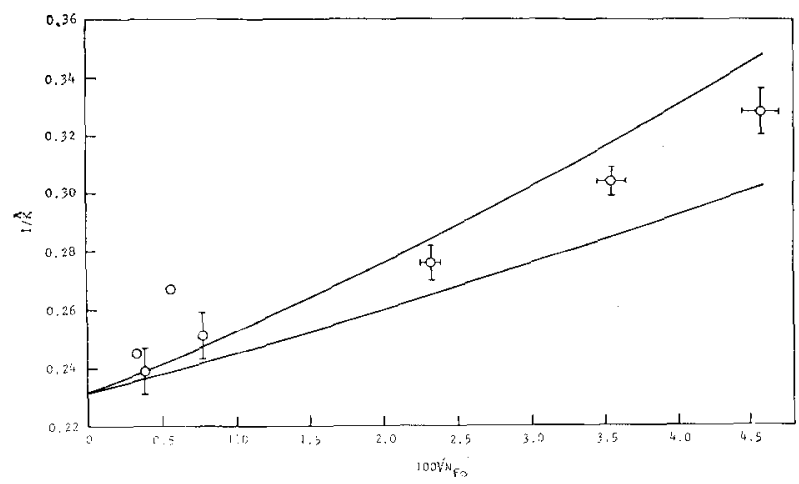

Fig. 12-Experimental data, with data bound lines calculated from heat-transfer analysis

thermocouple lag time, and response characteristics of the two thermocouples are closely matched.

Table 1 reports parameters and results for the specimens used in the experimentation. Thermal diffusivity values listed are taken from recent property compilations, and are felt to be accurate to 5 percent. Unloading times listed are measured from the strain-gage traces, and standard deviations calculated from the data are shown. Error limits shown for the Fourier number parameter are calculated from standard deviations associated with the thermal diffusivity and loading-time values. The stress levels shown are stress ranges at the plate locations, calculated from the strain-gage deflections. Values of $1 / \hat{K}$ calculated from the experimental temperature measurements are also shown, with associated standard deviations.

Results are presented in Figs. 12 and 13. In Fig. 12, upper and lower bounds from the analysis are shown, and experimental data listed in Table 1 are plotted, including data for the two specimens which failed to satisfy the thermocouple lag-time criterion. The effect of dissimilar thermocouple response at the two measurement locations is shown by these two data points. Here, thermocouple response at the hole location is slower than plate thermocouple response, and signal attenuation is greater for the hole thermocouple signal. Hence, these two data points lie above the upper bound. The valid data points, however, lie within the theoretical bounds, or have error limits which extend into the region predicted by the analysis.

The experimental technique for determining local stresses with thermal measurements is shown in
Fig. 13. The five valid data points are plotted, and the intercept of the straight line which best fits the experimental data is the inverse of the stressconcentration factor. The straight line shown minimizes the sum of squares of deviations of the dependent variable $1 / \hat{K}$ and assumes the independent variable is exact. A linear correlation of the data with the assumption that the $1 / \hat{K}$ values are exact and the Fourier number parameter subject to error gives the same value for the stress-concentration factor. The stress-concentration factor obtained from the analysis, with a 95 percent confidence range, is $4.3 \pm 0.18$. This agrees well with the theoretical value of 4.32 from Howland's analysis.

\section{Discussion}

The experimental technique can be extended to geometries where an analysis cannot be made. The technique in these cases consists in identifying the Fourier number for the geometry, making temperature-change measurements at various values of this parameter, and correlating the data in the manner shown in Fig. 13.

The similarity of stress distributions involved in various stress-concentration geometries allows this extension. This similarity is formalized in concepts such as the "equivalent ellipse"17 and the "law of the stress gradient." ${ }^{4}$ The analytical results from the model geometry also give confidence that the technique may be extended. The method is valid both for the shallow notch case, involving a two dimensional heat flow geometry, and the deep notch case, involving essentially a one-dimensional heatflow geometry.

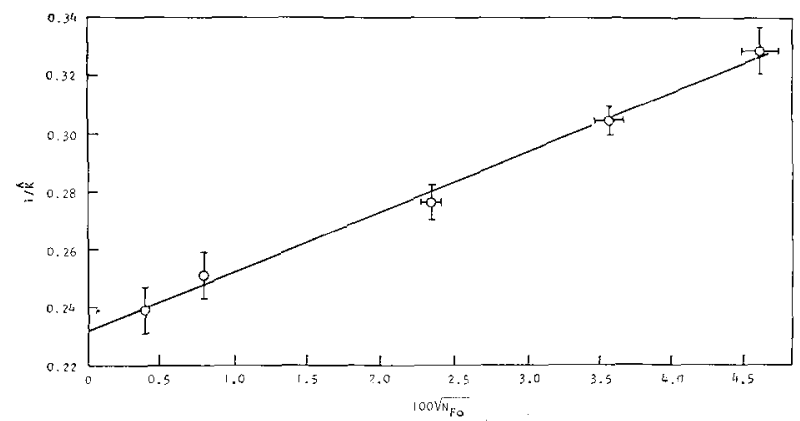

Fig. 13-Linear correlation of data involved in thermalmeasurement technique 
Pertinent applications of the technique extend to problems for which local rather than full-field stress measurement conveys adequate information. The effects of notch shape and depth on stress concentration are examples, as is the study of the interaction and superposition effects ${ }^{18}$ of holes and notches. Dynamic stress-concentration measurement is also a possible application of the thermalmeasurement technique. Here, useful results can be obtained only if thermocouples with microsecond-response characteristics are used, and the use of ultra-fine-wire thermocouples and carefully controlled welding techniques are indicated. $\mathrm{Re}-$ cent measurements of microsecond-response characteristics for thermocouples have been reported. ${ }^{19}$, 20 Finally, Dillon's measurements of thermoplastic temperature changes and the use of temperature-change measurements to study plastic deformations should be mentioned.21, 22 Extension of the thermal-measurement technique to these problems could provide valuable information for the experimental stress analyst.

\section{Acknowledgment}

The continuing assistance of R. J. Price, General Atomic, in this investigation is gratefully acknowledged.

\section{References}

1. Thomson, W. (Lord Kelvin), "Mathematical and Physical Papers," 1, Cambridge, London (1882).

2. Joule, J. P., "The Scientific Papers," 1, Physical Society of London, London, (1963).

3. Rocca, R., and Bever, M. B., "The Thermoelastic Effect," Trans. AIME $188,327-333(1950)$

4. Tichener, A. L., and Bever, M. B., "The Stored Energy of Cold Work," in "Progress in Metal Physics," 7, Pergamon Press, New York, N. Y., 247-338 (1958).

5. Dunlap, R. W., "A Study of Stress Measurements Using the Thermoelastic Effect," PhD Thesis, University of Michigan, Ann Arbor, Michigan (1966).

6. Coker, E. G., "On the Measurement of Stress by Thermal Methods, with an Account of Some Experiments on the Influence of Stress on the with an Account of Some Experiments on the Influence of Stress on the
Thermal Expansion of Metals," Trans. Roy. Soc. Edin., 48, 229-250

(1904).

7. Nadai, A., "Theory of Flow and Fracture of Solids," 2, McGrawHill, New York, N. Y., 2nd ed., 3-10 (1963).

8. Dillon, O. W., Jr., and Tauchert, T.R., "The Experimental Technique for Observing the Temperatures Due to the Coupled Thermoelastic Effect," Int. eJ. Solids Struc. 2, 385-391 (1966).

9. Howland, R. C. J., "On the Stresses in the Neighborhood of a Circular Hole in a Strip under Tension," Phil. Trans. Roy. Soc. London, 229A, 49-86 (1930).

10. Howland, R. C. J., and Stevenson, A.C., "Bi-Harmonic Analysis in a Perforated Strip," Phil. Trans. Roy. Soc. London, 232A, 155-222 (1934).

11. Frocht, M. M., "Photoelasticity," "John Wiley \& Sons, Inc., New York, N. Y., 1, (1941); 2, (1948).

12. Flynn, P. D., and Roll, A. A., "A Re-examination of Stresses in a Tension Bar with Symmetrical U-shaped Grooves," EXPERIMENTAL MECHANICS, 6, 93-98 (1966).

13. Durelli, A. J., Philipps, E. A., and Tsao, C. H., "Introduction to the Theoretical and Experimental Analysis of Stress and Strain," McGraw-Hill, New York, N. Y. (1958).

14. Neuber, H., "Theory of Notch Stresses," AEC-tr-4547, 2nd ed., Office of Technical Services, Dept. of Commerce, Washington 25, D. C. (1961).

15. Pearson, C. E., “Theoretical Elasticity," Harvard University Press, Cambridge, Mass. (1959).

16. Biot, M. A., "Linear Thermodynamics and the Mechanics of Solids," in "Proc. Third U. S. National Congress of Applied Mechanics"" ASME New York (1958).

17. Ross, D. S., and Qureshi, I. H., "Boundary Value Problems in Two-Dimensional Elasticity by Conducting Paper Analogue," J. Sci. Instrum., 40, 513-517 (1963).

18. Peterson, R. E., "The Interaction Effect of Neighboring Holes or Cavities, with Particular Reference to Pressure Vessels and Rocket Cases," J. Basic Eng., 87, 879-886 (1965).

19. Morrison, R. G., "Application of Miniature Intrinsic Thermo- couples for Reactor Transient Diagnostics," Trans. Am. Nucl. Soc., 8, 128-9 (1965).

20. Henning, C. D., and Parker, R., "Transient Response of an Intrinsic Thermocouple," J. Heat Transfer, 89, 146-154 (1967).

21. Dillon, O. W., Jr., "The Heat Generated During the Torsional Oscillations of Copper Tubes," Int. J. Solids Struc., 2, 181-204 (1966).

22. Dillon, O. W., Jr., "Coupled Thermoplasticity," J. Mech. Phys. Solids, 11, 21-33 (1963).

\section{APPENDIX}

\section{Temperature Changes During Linear Loading}

At the hole thermocouple location, the temperature change is described by eq (3) for an arbitrary loading process. The transformed equation corresponding to eq $(3)$ is

$$
\overline{\Delta T}(s)=s \bar{H}(s) \bar{F}(s)
$$

where $s$ is the Laplace transform parameter and $\bar{F}(s)$, for example, is the Laplace transform of the loading function $F(t)$ :

$$
\bar{F}(s)=\int_{0}^{\infty} e^{-s t} F(t) d t
$$

For the linear loading, the loading function is shown in Fig. 6 (upper curve), and is represented by the equation

$$
\begin{aligned}
F(t) & =t \Delta T_{\max } / t_{o}, \quad t \leq t_{o} \\
& =\Delta T_{\max }, \quad t \geq t_{o}
\end{aligned}
$$

The constant, $\Delta T_{\max }$, represents the maximum temperature change, corresponding to the maximum stress reached at the root of the hole:

$$
\Delta T_{\max }=-T_{a} \alpha \sigma_{\max } / \rho C p
$$

Hence, the transform $\vec{F}(s)$ is given as follows:

$$
\bar{F}(s)=\left[\Delta T_{\max } / t_{o} s^{2}\right]\left(1-e^{-s t_{0}}\right)
$$

Substituting eq (A-2) into eq (A-1) and inverting, the temperature change at the root of the hole for linear loading is

$$
\begin{aligned}
\Delta T(t) & =\left(\Delta T_{\max } / t_{o}\right) \int_{o}^{t} H(t) d t, \quad t \leq t_{o} \\
& =\left(\Delta T_{\max } / t_{o}\right) \int_{t-t_{o}}^{t} H(t) d t, \quad t \geq t_{o}
\end{aligned}
$$

The peak temperature change occurs at time $t_{o}$, and is

$$
\Delta T\left(t_{o}\right) \equiv \Delta T_{\mathrm{hole}}=\left(\Delta T_{\max } / t_{o}\right) \int_{o}^{t_{o}} H(t) d t
$$

At the plate thermocouple location, a uniform stress state exists, and no heat conduction occurs. The maximum stress during linear loading is $\sigma_{\max } / K$, where $K$ is the stress-concentration factor. The corresponding peak temperatures change is

$$
\Delta T_{\text {plate }}=\Delta T_{\max } / K
$$

The ratio of peak temperature changes at hole and plate is defined as $\hat{K}$, eq (4). The expression for $\hat{K}$ in linear loading, eq $(5)$, results from the substitution of eqs (A-4) and (A-5) into eq (4). 